\title{
Take the oral health challenge today
}

Johnson \& Johnson, the makers of LISTERINE, launched The Oral Health (OH) Challenge at the 2017 British Dental Conference and Exhibition, which was held in Manchester in May.

The $\mathrm{OH}$ Challenge is a simple survey tool created for dental healthcare professionals to test their knowledge in relation to soft tissue health and preventive care, and to identify any gaps in current professional knowledge.

$B D J$ readers are invited to take part in this important survey challenge. Your involvement and the results gathered will help create bespoke articles for the dental profession, designed to increase understanding of these all-important issues.
The $\mathrm{OH}$ Challenge is supported by Iain Chapple, Professor of Periodontology, Consultant in Restorative Dentistry and Head of the School of Dentistry at the University of Birmingham, and Julie Rosse, a past President of the British Society of Dental Hygiene and Therapy and practising hygienist.

The $\mathrm{OH}$ Challenge is available online for all dental health care professionals. Visit www.listerineprofessional.co.uk now to test your own knowledge and follow the supporting programme of evidence-based content that will be released in instalments over the course of the year.

For more information on LISTERINE visit www.listerineprofessional.co.uk.

\section{New toothpaste targets sensitivity}

GlaxoSmithKline Consumer Healthcare has announced an advancement in dental care with the launch of new Sensodyne Rapid Relief. The unique anhydrous formulation works rapidly to form a barrier over exposed dentine and reduce sensitivity in just 60 seconds [with twice daily brushing]. The new formulation builds on-going protection with every of dentine, helping to trap stannous ions which occlude the open ends of the dentine tubules. In vitro data shows that after just one application, stannous ions, the key sensitivity active ingredient in new Sensodyne Rapid Relief, can extend as much as $80 \mu \mathrm{m}$ deep into the tubules, providing rapid occlusion and a $127 \%$ reduction in the flow of fluid through the

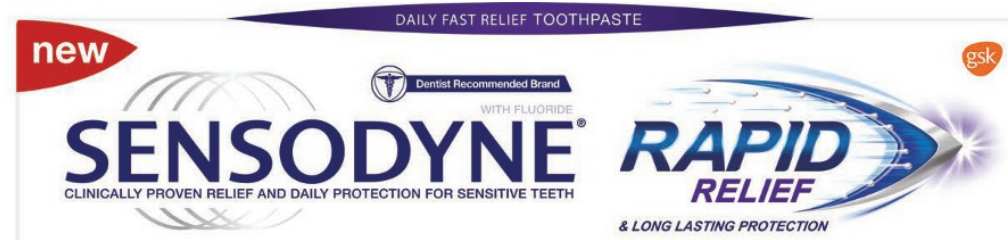

brush, supporting the long-term management of dentine hypersensitivity, a painful, chronic condition affecting as many as one in three people.

The use of GSK Consumer Healthcare's proprietary anhydrous formulation technology means that individual ingredients contained in the formulation, including stannous fluoride and bio-adhesive polymers, remain stable and ready for activation by water. On contact with saliva in the mouth, the facilitating polymer becomes adhesive, and is able to form a gel-like scaffold in the surface dentine tubules, compared to the previous stannous fluoride formulation. This robust occlusion is resistant to exposure to dietary acids, leading to clinically proven fast and long-lasting relief [with twice daily brushing] from the pain of dentine hypersensitivity.

GSK Consumer Healthcare intends to make dentine hypersensitivity an easily manageable condition over the long-term, building on their overall purpose to create a future where every life is sensitivity-free.

Sensodyne Rapid Relief is available in store now and is also available in Whitening.

\section{Shifting the paradigm}

Dentist Craig Parker and clinical dental technician (CDT) Jonathan Hughes recently copresented a lecture that explored new digital applications and materials for producing removable partial dentures (RPDs).

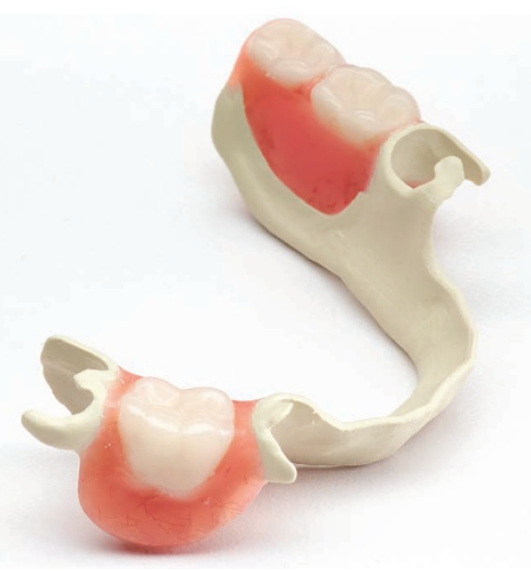

Located at the Dental Technology Showcase at Birmingham NEC, Craig and Jonathan introduced technicians to the revolutionary new Ultaire AKP, an innovative, highperformance, specially-formulated polymer for dental applications. They presented it as an alternative to metal for RPDs and highlighted the following attributes:

- Ultaire AKP is durable and does not distort

- Lightweight

- Ultaire AKP has high levels of conformity

- Its flexural properties allow engagement for deeper undercuts than metal

- It can be coloured

- Ultaire AKP is easy to trim and adjust

- Taste free - no metallic taste

- Technicians can produce a natural looking smile with discreet clasp designs

- Using Ultaire AKP eliminates the risk of metal sensitivity

- The material is similar to bone and patients like the feel of it.

Other key benefits discussed were that with an Ultaire AKP RPD frame, point loading is reduced; the frames have the incorporation of rests so it can be tooth borne and energy dissipated across the framework. There are thoughts this could limit bone loss.

If you were not able to attend the lecture you can still find out about Ultaire AKP from by contacting Solvay Dental 360 now. Visit www.solvaydental360.com. 